\title{
DETECTION OF SUBCLINICAL PAROXYSMAL ATRIAL FIBRILLATION AND ITS CORRELATION WITH CANDIDATE GENES IN PATIENTS WITH CRYPTOGENIC ISCHEMIC STROKE AND TIA
}

\author{
Petrovicova A ${ }^{1}$, Kurca E ${ }^{2}$, Andrasova $A^{3}$, Bernatova $\mathrm{J}^{4}$, Blasko ${ }^{3}$, Burjanivova $\mathrm{T}^{5}$, Duris \\ $\mathrm{T}^{4}$, Grendar $\mathbf{M}^{6}$, Hasilla $J^{7}$, Malicherova $\mathrm{B}^{5}$, Nosal $\mathrm{V}^{2}$, Obona $\mathrm{P}^{3}$, Patrovic $\mathrm{L}^{8}$, SivaK $\mathrm{S}^{2}$, \\ SNOPEK $\mathrm{P}^{7}$, SvetlosaK $\mathrm{M}^{9}$, VAHALA $\mathrm{P}^{\dagger 7}$, CieRNY $\mathrm{D}^{10}$
}

${ }^{1}$ Clinic of Neurology, Faculty Hospital Nitra, Špitálska 6, 94901 Nitra, Slovak Republic

${ }^{2}$ Clinic of Neurology, Jessenius Faculty of Medicine in Martin, Comenius University in Bratislava, and University Hospital Martin, Kollárova 2, 03659 Martin, Slovak Republic

${ }^{3}$ Cardiocenter Nitra, Špitálska 6, 94901 Nitra, Slovak Republic

${ }^{4}$ Clinic of Internal Medicine, Faculty Hospital Nové Zámky, Slovenská ulica 11 A

940 34, Nové Zámky 1, Slovak Republic

${ }^{5}$ Department of Molecular Biology and Genomics, Jessenius Faculty of Medicine in Martin,

Comenius University in Bratislava, Malá Hora 4C, 03601 Martin, Slovak Republic

${ }^{6}$ Bioinformatic centre, Biomedical Center Martin, Jessenius Faculty of Medicine in Martin,

Comenius University in Bratislava, Malá Hora 4C, 03601 Martin, Slovak Republic

${ }^{7}$ Clinic of Cardiology, Faculty Hospital Nitra, Špitálska 6, 94901 Nitra, Slovak Republic

${ }^{8}$ Jessenius diagnostic centre, Špitálska 557, 94901 Nitra, Slovak Republic

${ }^{9}$ Department of Arrhythmias and Cardiac Pacing, National Institute for Cardiovascular Diseases,

Pod Krásnou hôrkou 1, 83348 Bratislava 37, Slovak Republic

${ }^{10}$ Department of Clinical Biochemistry, Jessenius Faculty of Medicine in Martin, Comenius University in Bratislava, and University Hospital Martin, Kollárova 2, 03659 Martin, Slovak Republic

\section{A b s tract}

Introduction: Cardioembolic etiology is assumed to be the most frequent cause of cryptogenic strokes. The detection of subclinical paroxysmal atrial fibrillation $(\mathrm{AF})$ is important in the correct choice of preventive treatment. The aim of this prospective study was to detect the incidence of AF in patients with a cryptogenic stroke or transient ischemic attack (TIA) and to evaluate the association between the presence of AF and selected single-nucleotide polymorphisms (SNP).

Methods: Patients with a cryptogenic stroke/ TIA $(n=100)$ and a control group $(n=15)$ of volunteers without significant cardiovascular disease were included in the study during the period of 2014 to 2019. To detect AF they underwent 12 months of ECG monitoring using an implanted loop recorder (ILR). Genotyping for SNPs rs10033464, rs2200733, rs225132, and rs2106261 was performed by a high resolution melting analysis.

Results: We found AF to be present in 24 (24\%) patients with a cryptogenic stroke/TIA, versus no subjects in the control group. The SNPs rs2106261, rs2200733, rs225132, and rs10033464 were not found to be associated with $\mathrm{AF}$ in our study $(\mathrm{p}=0.240 ; 1.000 ; 0.887 ; 0.589)$. However, a weak trend for a higher frequency of rs2106261 risk allele A homozygotes was observed in the patients with $\mathrm{AF}$ compared to the patients without $\mathrm{AF}(0.416$ vs. 0.263, $\mathrm{p}=0.073$ ). Homozygotes for allele A of rs2106261 were also present in a significantly higher frequency in AF patients compared to the controls $(0.416$ vs. 0.133, $\mathrm{p}=0.012)$.

Conclusion: In our study paroxysmal AF was a probable etiological factor in $24 \%$ of patients with cryptogenic ischemic stroke / TIA during the 12 months of monitoring. The homozygous allele A of rs2106261 was identified to be the possible genetic risk factor of $\mathrm{AF}$, but this should be verified in larger cohorts.

The study has been registered at www.clinicaltrials.gov, identifier NCT02216370.

Key-words: transient ischemic attack, cryptogenic stroke, atrial fibrillation, implantable loop recorder, singlenucleotide polymorphisms

Corresponding author: Daniel Cierny, e-mail: daniel.cierny@uniba.sk

(C) 2021 Cierny D. et al.

This work is licensed under the Creative Commons Attribution-NonCommercial-NoDerivs 4.0 License (https://creativecommons.org/licenses/by-nc-nd/4.0/) 


\section{INTRODUCTION}

An ischemic stroke (IS) is a heterogeneous multi-factor polygenic disease with a high mortality rate and long-term consequences. (1) One of the known, important, and independent risk factors for IS is atrial fibrillation (AF). (2) Its early detection is of fundamental prognostic importance. Paroxysmal AF is often considered a probable cause of cryptogenic stroke. (3) The detection of subclinical paroxysmal AF in this case is difficult because it can be asymptomatic, mainly in older people, and traditional 12-lead or Holter ECGs have a low level of detection due to the limited length of the recording. In recent years the higher detection of $\mathrm{AF}$ is due to the introduction of long-term monitoring strategies. Non-stop long-term ECG monitoring using implantable devices is a better and clinically more effective technique than conventional monitoring, in particular following a cryptogenic stroke. In order to achieve maximum AF detection further factors should be identified in order to choose patients for a long-term monitoring. Greater attention is paid to genetic studies under the assumption that genetic tests might help define the subtype of a cryptogenic stroke. (4-8)

The aim of the work was to define the following in a group of patients with a cryptogenic stroke/ transient ischemic attack (TIA): 1) the presence of AF using an implantable ECG loop recorder (ILR); 2) the number of recurrences of stroke/ TIA during the following 12 months; 3) the presence of new ischemic lesions during a magnetic resonance (MRI) examination 12 months later; 4) whether there is a relation between newly-diagnosed AF in our group of Slovak patients and the find of selected single-nucleotide polymorphisms (SNPs) in literature associated with $\mathrm{AF}$ and cardioembolic infarction.

\section{METHODS}

\section{Characteristics of the groups}

One hundred patients with an acute ischemic stroke or TIA were included in the study. The patients were hospitalised within 72 hours from the onset of the ischemic stroke at the Clinic of Neurology of the Faculty Hospital in Nitra from 2014 to 2019 and they met the definition of cryptogenic etiology according to TOAST criteria (inclusion and exclusion criteria) during the screening period. Transient ischemic attack was defined as a temporary neurological deficit of vascular origin without acute ischemic MRI changes. The possible etiological factors of ischemic stroke were excluded in all patients by: CT and MR imaging of the brain and intra/extracranial arteries, hematological testing for thrombophilia, rheumatological tests for vasculitis, 14 days lasting 12-lead Holter ECG for detection of paroxysmal $\mathrm{AF}$, transthoracic and transoesophageal echocardiography for cardioembolism. The control group was made up of 15 volunteers without a significant cardiovascular and cerebrovascular disease adjusted by age and gender. The patients were monitored for 12 months. All patients and volunteers in the control group signed an informed consent form.

\section{Examinations}

The diagnosis of an ischemic stroke required consistent neuroimaging findings on CT and MRI. In control individuals MRI had not been performed before participating in the study. All subjects in patient and control groups in addition underwent a follow-up MRI once the monitoring was finished. Standard non-contrast CT and CT angiography of the aortic arch, cervical and intracranial arteries (SIEMENS Somatom Definition, dual source 2x128, Stellar upgrade, SIEMENS Somatom Edge 128), and brain MRI (SIEMENS Skyra 3.0T, SIEMENS Magnetom Avanto 1.5T, SIEMENS Magnetom Symphony 1.5T (protocol T2wTSE SAG + TRA, FLAIR T2wTIRM dark fluid - TRA, DWI - epi2ddiffusion - TRA + ADC, MRA-TOF3D multislab - TRA + MPR + MIP + VRT, CE imag.-i.v. inject. application + aqua flush 30ml T1w mpr ISO - TRA + MPR / COR + SAG) were carried out at the JESSENIUS diagnostics centre. The CT and MRI findings were evaluated by independent radiologists. 


\section{Implantation of a long-term ECG loop recorder}

The implantation of a ILR (REVEAL LINQ / XT, Medtronic Inc., Minneapolis, USA) was carried out within 6 months of the onset of the ischemic stroke/ TIA as arranged with a cardiologist in the following sites: Clinic of Cardiology of the Faculty Hospital in Nitra; Kardiocentrum Nitra s.r.o.; Department of Arrhythmology of the National Institute of Cardiovascular Diseases and the Clinic of Internal Medicine of the Faculty Hospital in Nové Zámky. AF was defined as an episode of irregular cardiac rhythm without the presence of a $\mathrm{P}$ wave, lasting for more than 2 minutes. The ECG output obtained from the ILR during check-ups was assessed by an arrhythmologist.

\section{Check-up examination}

During the final check-up examination after 12 months we assessed the AF finding, the result of the follow-up brain MRI examination, the recurrence of acute stroke, and a change in treatment in the secondary prevention of acute stroke. In cases where AF was detected by ILR in the course of the 12-month monitoring period the patients and the control subjects underwent an unplanned neurological check-up with a change from antiplatelet treatment to anticoagulation. An unplanned neurological check-up was also undergone in the case of a recurrence of acute stroke.

\section{Genetical analysis}

The genetic examination was carried out at the Department of Molecular Biology and Genomics, the Jessenius Faculty of Medicine in Martin and BioMed, Comenius University in Bratislava, in cooperation with the Department of Clinical Biochemistry, Jessenius Faculty of Medicine in Martin, Comenius University in Bratislava, and University Hospital Martin. Four SNPs were selected for genetic examination: rs10033464 (PITX2c gene), rs 2200733 (PITX2c gene), rs225132 (ERRF11 gene), and rs2106261 (ZFHX3 gene). The characteristics of the analysed SNPs are shown in Table 1.

Table 1 Selected single-nucleotide polymorphisms (SNP)

\begin{tabular}{|c|c|c|c|c|}
\hline SNP & Genetic locus & Chromosome & Major / risk allele & Susceptibility, population \\
\hline rs2106261 & ZFHX3 & $16 \mathrm{q} 22$ & $\mathrm{G} / \mathrm{A}$ & $\begin{array}{c}\text { AF, Europeans } \\
\approx 11 \% \text { Han Chinese }\end{array}$ \\
\hline rs2200733 & PITX2c & $4 \mathrm{q} 25$ & $\mathrm{C} / \mathrm{T}$ & $\mathrm{AF}$, Europeans \\
\hline rs225132 & ERRF11 & 1 & $\mathrm{~T} / \mathrm{G}$ & $\begin{array}{c}\text { All types of ischemic } \\
\text { stroke, coronary heart } \\
\text { disease, Europeans }\end{array}$ \\
\hline rs10033464 & PITX2c & $4 \mathrm{q} 25$ & $\mathrm{G} / \mathrm{T}$ & $\mathrm{AF}$, Europeans \\
\hline
\end{tabular}

Abbreviations: AF - atrial fibrillation

The samples of peripheral blood were taken and dispensed into $3 \mathrm{ml}$ tubes containing 5,4 mg of EDTA (Vacutest Kima S.r.1., Italy). DNA was isolated using DNeasy Blood \& Tissue Kit (Qiagen, Valencia, CA, USA). The concentration and purity of the DNA were determined using Nanodrop (ND-1000, NanoDrop Technologies, Wilmington, DE). All samples were diluted in sterile distilled water to a $25-50 \mathrm{ng} / \mu \mathrm{l}$ final concentration. PCR amplification and genotyping was performed by a High Resolution Melting (HRM) analysis using the Roche LightCycler® 480 Instrument (Roche, USA). The reaction volume consisted of $10 \mu \mathrm{l}$ of LC480 HRM Master Mix, 2,4 $\mu \mathrm{l}$ of $\mathrm{MgCl}_{2}$ (25 mM), $2 \mu \mathrm{l}$ of genomic DNA sample (25-50 ng), 
and $0.4 \mu \mathrm{l}$ of $10 \mu \mathrm{M}$ primers (Ecoli, Czech Republic) filled up to a total volume of $20 \mu \mathrm{l}$ by redistilled water. The oligonucleotide primers were designed with the online uDesignSM application (www.dna.utah.edu/udesign/index.html). The primer sequences are shown in Table 2. The reaction conditions for rs 10033464 and $\mathrm{rs} 2106261$ were: $95^{\circ} \mathrm{C}$ for $10 \mathrm{minu}-$ tes, $\left(95^{\circ} \mathrm{C}\right.$ for 10 seconds, $66^{\circ} \mathrm{C}$ for 15 seconds, $72^{\circ} \mathrm{C}$ for 10 seconds) $\mathrm{x} 45$ cycles; $72^{\circ} \mathrm{C}$ for 5 minutes. Heteroduplex PCR was performed at $95^{\circ} \mathrm{C}$ for 1 minute, $40^{\circ} \mathrm{C}$ for 1 minute; and c) HRM: 65 to $95^{\circ} \mathrm{C}$ with a ramp rate set to $0.02^{\circ} \mathrm{C} /$ second and 25 acquisitions $/{ }^{\circ} \mathrm{C}$. The reaction conditions for $\operatorname{rs} 225132$ and rs 2200733 were: $95^{\circ} \mathrm{C}$ for 10 minutes, $\left(95^{\circ} \mathrm{C}\right.$ for 10 seconds, $60^{\circ} \mathrm{C}$ for 15 seconds, $72^{\circ} \mathrm{C}$ for 10 seconds) $\mathrm{x} 45$ cycles; $72^{\circ} \mathrm{C}$ for 5 minutes. Heteroduplex PCR was performed at $95^{\circ} \mathrm{C}$ for 1 minute, $40^{\circ} \mathrm{C}$ for 1 minute. HRM analysis was performed at 65 to $95^{\circ} \mathrm{C}$ with a ramp rate set to $0.02^{\circ} \mathrm{C} /$ second and 25 acquisitions $/{ }^{\circ} \mathrm{C}$. To verify the quality of genotype determination several samples from each SNP were bi-directionally sequenced by Sanger sequencing. PCR fragments were purified with ExoSap-IT (Applied Biosystems, Foster City, CA, USA). Sequencing of SNPs was performed using BigDye Terminator v3.1 Cycle Sequencing kit (Applied Biosystems, Ca, USA) on the ABI PRISM 3130x1 automatic sequencer (Applied Biosystems, CA, USA). Sequence data analysis was carried out by using Chromas software (http://www.technely-sium.com.au/chromas.html).

Table 2 Primers used for HRM

\begin{tabular}{|l|l|}
\hline \multirow{2}{*}{ SNP } & Primer sequence \\
\hline \multirow{2}{*}{ s 2106261} & F 5 CACAGATAGAGCTCGTCCAGAGAATTG \\
\cline { 2 - 3 } & R 5' GCCACTTGGATATTTTAATGGATGGTT \\
\hline \multirow{2}{*}{ s 2200733 } & F 5' GTGGTACTTGGGTTTTGATTTTGA \\
\cline { 2 - 2 } & R 5' ACTACCTTAAATATTACCTGTTCTAATTTTCTC \\
\hline \multirow{2}{*}{ rs225132 } & F 5' TCAGTGAAGAGGTGGTGAATGTAGGAAT \\
\cline { 2 - 2 } & R 5' ACCATGTGCCATGAATGCAA \\
\hline \multirow{2}{*}{ rs10033464 } & F 5' CAATTTAAAATTTTCTTTTTTTACATTGTTAGAGTCAAGAA \\
\cline { 2 - 2 } & R 5' CTCAGAGCTTGATGAAAGCACT \\
\hline
\end{tabular}

Abbreviations: AF - atrial fibrillation

\section{Statistics}

We used common methods of descriptive statistics. We evaluated the association between $\mathrm{AF}$ and the other categorical variable, SNPs, using a contingency table. The zero hypothesis on the non-existence of an association between atrial fibrillation and another categorical variable (SNP) was tested using a Pearson's Chi-squared test with Yates' continuity correction and a Cochran-Armitage trend test. All the data analyses were done in R [15], ver. 3.5.2.

\section{Registration of the study}

The study has been registered at www.clinicaltrials.gov under the identifier NCT02216370. 


\section{RESULTS}

\section{Demographic data}

The mean age of the subjects in the patient group was 70.1 years (28-87) vs. 75.4 years (35-90) in the control group. The ratio of women in the patient group was 30\% (30) vs. 66.6\% (10) in the control group. 90\% (90) of patients had experienced a cryptogenic ischemic stroke with a moderate neurological deficit; the median NIHSS was 6.9 (0-15). The ratio of TIA in the patient group was $10 \%(10)$. The subjects in the patient group showed an average CHA2DS2-VASc score of $3(2-5)$ and $1.4(0-3)$ in the control group. A total of $68(68 \%)$ of the subjects in the patient group had an arterial hypertension in their personal history (18 (75\%) patients with detected AF vs. 9 (60\%) of the volunteers in the control group). In the patient group a total of $14(14 \%)$ patients had diabetes mellitus, of which $1(4.2 \%)$ patient had detected AF vs. 2 (13.3\%) volunteers in the control group. Clinical characteristics of the involved individuals are summarised in Table 3.

Table 3 Clinical characteristics of involved individuals

\begin{tabular}{|l|c|c|c|}
\hline Characteristics & $\begin{array}{c}\text { With AF } \\
(n=24)\end{array}$ & $\begin{array}{c}\text { Without AF } \\
(n=76)\end{array}$ & $\begin{array}{c}\text { Controls } \\
(n=15)\end{array}$ \\
\hline Age (median) & 64.29 & 62.59 & 75.46 \\
\hline Women \% (n) & $29.16(7)$ & $43.42(23)$ & $66.6(10)$ \\
\hline Cryptogenic stroke & 22 & 68 & 0 \\
\hline TIA & 2 & 8 & 0 \\
\hline NIHSS median (range) & $6.6(0-13)$ & $7.1(0-15)$ & 0 \\
\hline CHA ${ }_{2}$ DS ${ }_{2}$ VASc median (range) & $3(2-4)$ & $3(2-5)$ & $1.4(0-3)$ \\
\hline Hypertension \% (n) & $75(18)$ & $65.8(50)$ & $60(9)$ \\
\hline Diabetes mellitus \% (n) & $4.17(1)$ & $17.11(13)$ & $13.3(2)$ \\
\hline
\end{tabular}

Abbreviations: TIA - Transient ischemic attack, AF - Atrial fibrillation, NIHSS - The National Institutes of Health Stroke Scale, $\mathrm{n}$ - number

Detection of atrial fibrillation, recurrence of acute ischemic stroke, and change of treatment AF was confirmed in $24 \%(n=24)$ of the patients out of 100 in the patient group. The mean time interval between ILR and AF was 3.9 months (1-11 months). AF was not confirmed in any volunteer in the control group. Within the course of the 12-month monitoring period no subject in the patient nor control groups experienced a clinical or asymptomatic recurrence of an ischemic stroke or a systemic embolism. After the 12 months of monitoring MRI did not reveal any new ischemic brain lesions in the patient and control groups. In the patient group with $\mathrm{AF}(\mathrm{n}=24)$ the antiplatelet therapy was changed immediately to anti-coagulants (Table 4). 
Table 4 Detection of atrial fibrillation, change in MRI finding, change in preventive treatment after 12 months

\begin{tabular}{|c|c|c|c|}
\hline \multirow[t]{2}{*}{ Characteristics } & \multicolumn{2}{|c|}{$\begin{array}{l}\text { Patient group } \\
(n=100)\end{array}$} & \multirow{2}{*}{$\begin{array}{c}\begin{array}{c}\text { Control group } \\
(n=15)\end{array} \\
\begin{array}{c}\text { Without } A F \\
(n=15)\end{array}\end{array}$} \\
\hline & $\begin{array}{c}\text { With } A F \\
(n=24)\end{array}$ & $\begin{array}{c}\text { Without AF } \\
(n=76)\end{array}$ & \\
\hline Implantation of ICM\% (n) & $100(24)$ & $100(76)$ & $100(15)$ \\
\hline Time before the implantation of ICM ( $m$ ) & $2.5(1-4)$ & $1-6$ & $1-6$ \\
\hline Time preceding detection of $A F(m)$ & $3.9(1-11)$ & 0 & 0 \\
\hline Recurrence of IS /SE & 0 & 0 & 0 \\
\hline New MRI ischemic lesions after $12 m \%$ (n) & $0(0)$ & $0(0)$ & $0(0)$ \\
\hline Change in preventive treatment $\%$ ( $n$ ) & $100(24)$ & $0(0)$ & $0(0)$ \\
\hline
\end{tabular}

Abbreviations: IS = ischemic stroke, $\mathrm{SE}=$ systemic embolisation

\section{Analysis of the association of selected SNPs with AF in the patient group}

The genotype frequencies of the analysed SNPs in the subgroups of patients and in the controls are shown in Table 5.

SNP rs2106261 had no statistically significant association with AF ( $\mathrm{p}=0.240$ ). However, a weak trend for a higher frequency of homozygous form of rs2106261 risk allele A in patients with $\mathrm{AF}$ compared to the patients without AF was observed (41.6\% vs. 26.3\%, $\mathrm{p}=0.073)$. Allele $\mathrm{G}$ of rs2106261 was present in 14 (58.4\%) patients with AF vs. 56 (73.7\%) without AF. The trend for association of rs2106261 with AF was also found when compared the patients with AF to the controls ( $p=0.058$ ). The AA homozygotes were 10 patients with $\mathrm{AF}$ compared to 2 individuals in the control group and the difference was statistically significant (41.6\% vs. 13.3\%; $\mathrm{p}=0.012$ ). Allele $\mathrm{G}$ of rs2106261 was found in $14(58.4 \%) \mathrm{pa}-$ tients with AF vs. 13 (86.7\%) volunteers in the control group.

We found a weak trend for the association of rs2106261 with AF ( $p=0.076$ ). Homozygotes for allele A of rs2106261 were present in a significantly higher frequency in AF patients compared to the controls $(41.6 \%$ vs. $13.3 \%, p=0.012)$.

A positive finding of rs2200733 risk allele T homozygotes was observed in 5 (21\%) patients with AF vs. 15 (20\%) patients without AF. Allele C of rs2200733 was found in 19 (79\%) patients with AF vs. 61 (80\%) patients without AF. A statistically significant association of rs2200733 with AF was not found $(\mathrm{p}=1)$. When comparing patients with AF to the control group, homozygotes for risk allele T of rs2200733 were in 5 (20.8\%) patients vs. 3 (20\%) volunteers in the control group. Allele T of rs2200733 in homozygous form was present in $19(79.8 \%)$ patients with AF vs. 12 (80\%) volunteers in the control group. In patients with AF compared to the control group, rs2200733 did not show any statistically significant association with AF ( $\mathrm{p}=0.945)$.

Homozygotes of rs225132 minor allele G were 5 (20.8\%) patients with AF vs. 19 (25\%) patients without AF. Ancestral allele T of rs225132 was found in 19 (79.2\%) patients with AF vs. 57 (75\%) patients without AF. A statistically significant association of rs225132 with $\mathrm{AF}$ was not confirmed ( $\mathrm{p}=0.887$ ). When comparing patients with $\mathrm{AF}$ to the control group, homozygous allele $\mathrm{G}$ of rs225132 was found in $5(20.8 \%)$ patients vs. 5 (33.3\%) volunteers in the control group. Allele T of rs225132 was confirmed in 19 (79.2\%) patients with AF 


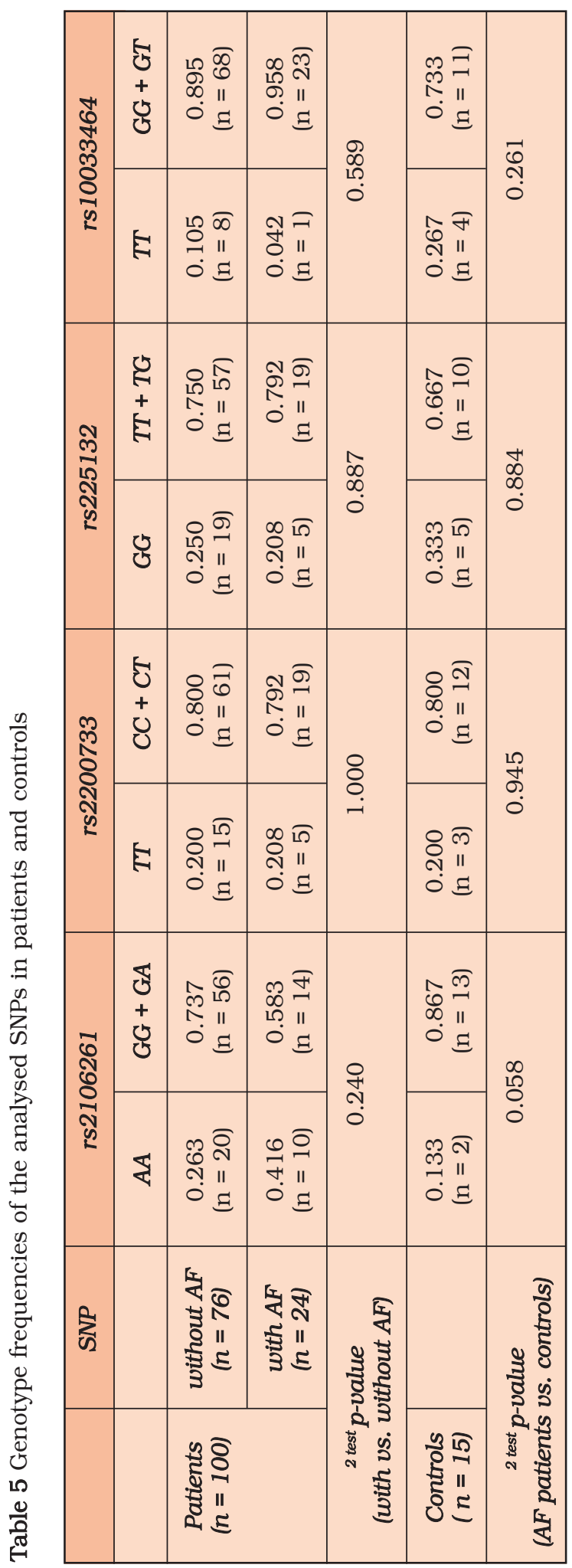


vs. 10 (66.4\%) healthy volunteers. A statistically significant association of $r s 225132$ with $\mathrm{AF}$ was also not shown when patients with AF were compared to the controls $(\mathrm{p}=0.884)$.

Homozygous risk allele $\mathrm{T}$ of $\mathrm{rs} 10033464$ was present in 1 (4.2\%) patient with AF vs. 8 (10.5\%) patients without AF. Allele G of rs 10033464 was found in 23 (95.8\%) patients with AF vs. 68 (89.4\%) patients without AF. A statistically significant association of rs 10033464 with $\mathrm{AF}$ was not confirmed $(\mathrm{p}=0.589)$. When comparing patients with $\mathrm{AF}$ to the control group, homozygous rs 10033464 risk allele $\mathrm{T}$ was present in 1 (4.2\%) patient vs. 4 (26.7\%) volunteers in the control group, and the difference was statistically significant $(\mathrm{p}=0.047)$. Ancestral allele G of rs10033464 was found in 23 (95.8\%) patients vs. 11 (73.3\%) volunteers in the control group.

\section{DISCUSSION}

In our study we found that paroxysmal AF was a probable etiological factor in $24 \%$ of patients with cryptogenic ischemic stroke/ TIA. In the control individuals paroxysmal AF was not present during the 12 months of monitoring. In the randomised control CRYSTAL-AF clinical study the AF detection after 12 months was lower compared to our findings, 12.24\% versus $24 \%$. In the above-mentioned study a 30.0\% detection rate was only achieved after 36 months of monitoring. (9) In the same manner, a lower detection rate of AF over an average monitoring period of 365 days, compared to our results, was found by Marks et al. (2019) in a retrospective study based on real practice. Using an ILR the authors detected a subclinical AF in 35 patients (19.6\%) in a group of 178 patients with cryptogenic ischemic stroke. (10) The detection rate of $\mathrm{AF}$ in our patients approximately correlates with the data of the meta-analysis of 28 studies processed by Tsivgoulis et al. (2019) in patients with cryptogenic ischemic stroke and embolic stroke of underdetermined source (ESUS) (of which 10 studies monitored patients from 6 to 12 months). (11) The cumulative AF detection rate in patients with ILR was $26 \%$ of the above-mentioned meta-analysis. Depending on the duration of monitoring, the authors found significant differences in the AF detection rates (p <0.001) ( <6 months: 5\%; $\geq 6$ and $\leq 12$ months: $21 \%$; >12 and <24 months: $26 \%$ $>24$ months: 34\%). They did not confirm any association with other patient characteristics, such as the subtype of ischemic stroke (cryptogenic vs. ESUS), or the time from the beginning of the ischemic stroke to the implantation of the ILR. These findings can be extrapolated to our research. The time of ILR implantation in all our patients within 6 months of the ischemic stroke/ TIA, which was needed for the organisation, performance of examinations and assessment, did not affect the AF detection rate.

In all 24 patients (100\%) with $\mathrm{AF}$ an antiplatelet therapy was changed to anticoagulation following the recommendations of the ESC (European Society of Cardiology, 2016). (12) We noted no recurrence of ischemic stroke either in the patients or in the control group over the monitored period (12 months), probably due to a short period and an appropriate change of treatment. Unlike our findings, a population study in Oxfordshire, United Kingdom, performed by $\mathrm{Li}$ et al. (2015) in patients with a first TIA or cryptogenic ischemic stroke revealed that a 10 -year risk of death was $46 \%$, vascular death was $15 \%$, and the recurrence of any form of ischemic stroke was 32\%. The risk of recurrence of ischemic stroke/ TIA in this study was lower compared to patients with a cardioembolic etiology ( $\mathrm{p}=0.003)$. (13) We can only speculate whether the individual efficacy of antiplatelet therapy, possibly depending on p-selectin expression, could be related to a recurrency of ischemic stroke/TIA (14).

A follow-up brain MRI after the 12 months of monitoring did not reveal new asymptomatic ischemic lesions in the patient and control groups. Studies dealing with the MRI detection of new ischemic lesions after a cryptogenic ischemic stroke focus more on proof of low-level symptomatic carotid stenosis, atherosclerosis of small intracranial arteries, or foramen ovale patens as etiological causes. On the other hand, Bal et al. (2012) in a group of 333 
(48\%) patients with cryptogenic ischemic stroke, out of the total number of patients with a mild stroke/ TIA (National Institutes of Health Stroke Scale score NIHSS $\leq 3$ ), found that $6.6 \%$ of patients had new lesions at the 30-day and $14.5 \%$ of patients at the 90-day MRI follow-up. (15) This population showed a high rate of silent radiological recurrence, which points to the disease activity. However, the study does not mention the type of therapy administered as part of the secondary prevention of ischemic stroke. In our study the patients had a higher NIHSS score 7 , they were given an antiplatelet therapy and an anticoagulation therapy after the finding of $\mathrm{AF}$. The relation between $\mathrm{AF}$ and brain lesions caused by a silent cerebral infarction (SCI) at an undefined time, diagnosed by MRI, has been examined in several studies. For example, the Framingham Offspring Study (Das et al., 2008) found a higher risk of SCI in patients with AF (OR 2.16). (16) On the contrary, Gaita et al. (2013) found white matter lesions in $92 \%$ of patients with persistent $\mathrm{AF}$, in $89 \%$ of patients with paroxysmal AF, and in $46 \%$ of patients without AF. A relation between SCI prevalence and the duration of $\mathrm{AF}$ was not found. (17)

Regarding the genetic analysis, in the case of rs2106261, we did not confirm a statistically significant association with $\mathrm{AF}(\mathrm{p}=0.240)$. However, we found a slight significant trend towards a higher frequency of rs2106261 AA homozygotes in patients with AF compared to the control individuals $(p=0.076)$. Interestingly, a significantly higher frequency of AA homozygotes was also present in patients with AF when compared to the controls $(p=0.012)$. Our results can be interpreted in accordance with the published data. Benjamin et al. (2009) in the meta-analysis of GWAS in patients with AF identified of a new susceptibility locus $Z F H X 3$, rs2106261 (RR $\left.=1.19 ; \mathrm{p}=2.3 \times 10^{-7}\right)$. (4) These findings were replicated in a cohort study of Psaty et al. (2009) from the German AF Network $\left(\mathrm{OR}=1.44 ; \mathrm{p}=1.6 \times 10^{-11}\right.$; combined $\mathrm{RR}=1.25$; combined $\left.\mathrm{p}=1.8 \times 10^{-15}\right)$. (5) It is interesting to note that the majority of available publications that identified the association of this SNP variant with AF mainly studied in Asian population by Li et al. (2011) and Zaw et al. (2017). (18, 19) In our study the rs2200733, rs225132, and rs10033464 did not show any significant association with AF. Our findings contradict Henninger et al. (2016) who found that patients with a cardioembolic stroke have a high-risk genetic profile, even despite age and numerous clinical co-morbidities. (20) Pulit et al. (2018) found that a polygenic risk score of AF explains $\sim 20 \%$ of the hereditary component of the cardioembolic risk of ischemic stroke. (21) Contrary to our findings, Gretarsdottir et al. (2008) and Gudbjartsson et al. (2009) consider the variant rs2200733 as one of the most important genetic polymorphisms associated with AF and cardioembolic stroke. $(6,7)$ This finding has been consistently replicated and validated in several cohorts of patients with a statistical significance of $\mathrm{p}<0.05$, in European and Asian populations by Wnuk et al. (2011), Viviani et al. (2008), Cao et al. (2013), and Sun et al. (2016). (22-25) The published information concerning rs10033464 is inconsistent and our negative findings indirectly and partially match the data in literature. Lemmens et al. (2010) found that rs 10033464 is only weakly associated with $\mathrm{AF}$ and this variant has demonstrated no relation to ischemic stroke. (26) However, in an isolated population of patients in Iceland, Gretarsdottir et al. (2008) reported a strong association between rs 10033464 risk allele $\mathrm{T}$ and cardioembolic stroke $\left(\mathrm{OR}=1.27 ; \mathrm{p}=6.1 \times 10^{-4}\right)$. (6) A strong association between $r$ s 10033464 and $\mathrm{AF}$ was also confirmed by Kääb et al. (2009) in cohorts of patients of European origin: the Framingham Heart Study (327 AF patients and 2006 controls, OR $=1.34$; 95\% CI 1.03-1.75; $\mathrm{p}=0.031$ ), the German AF Network (1715 patients with AF and 4073 controls, OR = 1.30; 95\% CI 1.13-1.51; $p=0.0002)$, and Rotterdam Study (910 AF patients and 5496 controls, $\mathrm{OR}=1.17 ; 95 \%$ CI 1.13-1.51; $0.99-1.38, \mathrm{p}=0.07)$. (8) On the other hand, the association of rs 10033464 with AF was not found in Vanderbilt AF Registry study that was performed in 556 patients with $\mathrm{AF}$ and 598 controls without $\mathrm{AF}(\mathrm{OR}=1.16$; 95\% CI 0.86-1.56; $\mathrm{p}=0.33$ ). (8) Interestingly, the autosomal dominant loci in ankyrin B protein 2 gene on chromosome 4 q25 were showed to be associated also with ventricular arrythmias, particularly long QT syndrome. (27) 
In the only publication dealing with rs225132 in context of AF, Traylor et al. (2012) (METASTROKE Collaboration) identified an association between the rs225132 variant and $\mathrm{AF}$ in patients with ischemic strokes $\left(\mathrm{p}=6.3 \times 10^{-8}\right.$ a $\left.5.9 \times 10^{-8}\right)$, and this is not in concordance with our findings. (28)

\section{CONCLUSION}

In our study we identified the high sensitivity of long-term ECG monitoring using an ILR for detecting subclinical paroxysmal $\mathrm{AF}$ as a possible etiological factor of cryptogenic ischemic stroke/TIA comparable to the published sources. In the majority of our patients $\mathrm{AF}$ would probably not have been detected at all under a shorter ECG monitoring. The causal relationship between the detected $\mathrm{AF}$ and ischemic stroke will require a further analysis. A neurogenic type of AF known as AFDAS (atrial fibrillation detected after stroke), which is triggered by an acute ischemic brain lesion, could explain the time correlation between $\mathrm{AF}$ and stroke in some of our patients.

One limitation of the study could be the selection of patients using the TOAST classification that is currently being shown to be insufficient, in particular for defining subgroups of patients with suspected subclinical AF. The selection of patients currently requires a close multidisciplinary (neurology-radiology-cardiology) cooperation, the inclusion of new diagnostic procedures, markers, predictive risk scores that were not known at the time when the methodology for this study was being prepared, or were not routinely carried out. Due to a relatively small number of included patients and control subjects, defining a causal relation between paroxysmal AF detected after ischemic stroke (AFDAS) might appear problematic and will require a further sub-analysis.

For genetic testing we chose four well-known single nucleotide polymorphisms possibly associated with AF - rs10033464 in PITX2c gene, rs2200733 in PITX2c gene, rs225132 in ERRF11 gene, and rs2106261 in ZFHX3 gene. None of these polymorphisms was shown to be significantly associated with $\mathrm{AF}$ in our study. However, in patients with cryptogenic ischemic stroke/ TIA a positive trend for the association of rs2106261 allele A homozygotes with $\mathrm{AF}$ was observed. This insignificant direct correlation may relate to the above-mentioned neurogenic subtype AFDAS which we did not analyse in our study. The possible confounding factor of these findings can be a relatively small cohort of examined individuals, which was due to the financial limitations of a study, especially by the number of available implantable ECG loop recorders. At present, however, 150 candidate genes have been identified for AF. Their variants could lead to a structural transformation of the heart in the form of atrial cardiomyopathy during intra-uterine development, or induced by stress in adulthood. For this reason, to confirm our findings and to clarify the exact mechanisms of involvement of candidate SNPs in AF and cryptogenic ischemic stroke, the future genetic and mainly functional studies including a whole-genome sequencing analysis in larger cohorts of individuals in different populations are necessary. We assume that including a genomic approach in the management of patients with cryptogenic ischemic stroke/ TIA might offer a narrower selection of patients for the implantation of a financially costly ILR and an earlier onset of targeted preventive treatment.

\section{REFERENCES}

1. Boehme AK, Esenwa C, Elkind MSV (2017) Stroke Risk Factors, Genetics, and Prevention. Circ Res $2017 ; 120: 472-495$.

2. Wolf PA, Abbott RD, Kannel WB Atrial fibrillation as an independent risk factor for stroke: the Framingham Study. Stroke 1991; 22:983-988. 
3. Sanna T, Diener H-C, Passman RS et al. Cryptogenic Stroke and Underlying Atrial Fibrillation. N Engl J Med 2014; 370:2478-2486.

4. Benjamin EJ, Rice KM, Arking DE et al. Variants in ZFHX3 are associated with atrial fibrillation in individuals of European ancestry. Nat Genet 2009; 41:879-81.

5. Psaty BM, O’Donnell CJ, Gudnason V et al. Cohorts for Heart and Aging Research in Genomic Epidemiology (CHARGE) Consortium: Design of prospective meta-analyses of genome-wide association studies from 5 cohorts. Circ Cardiovasc Genet 2 2009; 73-80.

6. Gretarsdottir S, Thorleifsson G, Manolescu A et al. Risk variants for atrial fibrillation on chromosome 4q25 associate with ischemic stroke. Ann Neurol 2008; 64:402-9.

7. Gudbjartsson DF, Holm H, Gretarsdottir S et al. A sequence variant in ZFHX3 on 16q22 associates with atrial fibrillation and ischemic stroke. Nat Genet 2009; 41:876-8.

8. Kääb S, Darbar D, van Noord C et al. Large scale replication and meta-analysis of variants on chromosome 4q25 associated with atrial fibrillation. Eur Heart J 2009; 30:813-9.

9. Sanna T, Diener H-C, Passman RS et al. Cryptogenic stroke and underlying atrial fibrillation. N Engl J Med 2014; 370:2478-86.

10. Marks D, Ho R, Nathan K et al. Abstract 17142: A Real-world Experience For Implantable Loop Recorder Monitoring To Detect Subclinical Atrial Fibrillation In Patients With Cryptogenic Stroke. Circulation 2019; 140:A17142-A17142.

11. Tsivgoulis G, Katsanos AH, Köhrmann M et al. Duration of Implantable Cardiac Monitoring and Detection of Atrial Fibrillation in Ischemic Stroke Patients: A Systematic Review and MetaAnalysis. J stroke 2019; 21:302-311.

12. Kirchhof P, Benussi S, Kotecha D et al. 2016 ESC Guidelines for the management of atrial fibrillation developed in collaboration with EACTS. Eur J Cardiothorac Surg 2016; 50:e1-e88.

13. Li L, Yiin GS, Geraghty OC et al. Incidence, outcome, risk factors, and long-term prognosis of cryptogenic transient ischaemic attack and ischaemic stroke: a population-based study. Lancet Neurol 2015; 14:903-913.

14. Kalmarova K, Kurca E, Nosal V et al. Measurement of platelet p-selectin expression by flow cytometry in patients with acute ischemic stroke. Acta Medica Martiniana 2018; 18(1):14-20.

15. Bal S, Patel SK, Almekhlafi M, Modi J, Demchuk AM, Coutts SB. High rate of magnetic resonance imaging stroke recurrence in cryptogenic transient ischemic attack and minor stroke patients. Stroke 2012; 43:3387-8 .

16. Das RR, Seshadri S, Beiser AS et al. Prevalence and correlates of silent cerebral infarcts in the Framingham offspring study. Stroke 2008; 39:2929-35.

17. Gaita F, Corsinovi L, Anselmino M et al. Prevalence of silent cerebral ischemia in paroxysmal and persistent atrial fibrillation and correlation with cognitive function. J Am Coll Cardiol 2013; 62:1990-1997.

18. Li C, Wang F, Yang Y et al. Significant association of SNP rs2106261 in the ZFHX3 gene with atrial fibrillation in a Chinese Han GeneID population. Hum Genet 2018; 129:239-46.

19. Zaw KTT, Sato N, Ikeda S et al. Association of ZFHX3 gene variation with atrial fibrillation, cerebral infarction, and lung thromboembolism: An autopsy study. J Cardiol 2017; 70:180-184.

20. Henninger N, Goddeau RP, Karmarkar A, Helenius J, McManus DD. Atrial Fibrillation Is Associated With a Worse 90-Day Outcome Than Other Cardioembolic Stroke Subtypes. Stroke 2016; 47:1486-92.

21. Pulit SL, Weng L-C, McArdle PF et al. Atrial fibrillation genetic risk differentiates cardioembolic stroke from other stroke subtypes. Neurol Genet 2018; 4:e293.

22. Wnuk M, Pera J, Jagiełła $J$ et al. The rs2200733 variant on chromosome 4q25 is a risk factor for cardioembolic stroke related to atrial fibrillation in Polish patients. Neurol Neurochir Pol 2011; 45:148-52.

23. Viviani Anselmi C, Novelli V, Roncarati R et al. Association of rs2200733 at 4q25 with atrial flutter/fibrillation diseases in an Italian population. Heart 2008; 94:1394-6.

24. Cao Y, Ma F, Wang Y, Wang DW, Ding H. Rs2200733 and rs10033464 on chromosome 4q25 confer risk of cardioembolic stroke: an updated meta-analysis. Mol Biol Rep 2013; 40:5977-85. 
25. Sun L, Zhang Z, Xu J, Xu G, Liu X. Chromosome 4q25 Variants rs2200733, rs 10033464, and rs1906591 Contribute to Ischemic Stroke Risk. Mol Neurobiol 2016; 53:3882-3890.

26. Lemmens R, Buysschaert I, Geelen V et al. The association of the 4q25 susceptibility variant for atrial fibrillation with stroke is limited to stroke of cardioembolic etiology. Stroke 2010; 41:1850-7.

27. Kujaník Š. Ventricular arrhytmias and genetics. Acta Medica Martiniana 2007; 7(1):3-9.

28. Traylor M, Farrall M, Holliday EG et al. Genetic risk factors for ischaemic stroke and its subtypes (the METASTROKE collaboration): a meta-analysis of genome-wide association studies. Lancet Neurol 2012; 11:951-62.

\section{Acknowledgements}

The authors would like to thank the patients with cryptogenic ischemic stroke and TIA and healthy control individuals for making this study possible.

Received: April, 4, 2021

Accepted: May, 7, 2021 